\title{
Article \\ Observer-Based Fuzzy Controller Design for Nonlinear Discrete-Time Singular Systems via Proportional Derivative Feedback Scheme
}

\author{
Wen-Jer Chang *D, Ming-Hsuan Tsai and Chin-Lin Pen \\ Department of Marine Engineering, National Taiwan Ocean University, Keelung 202, Taiwan; \\ g0046d011@gmail.com (M.-H.T.); pilotbhp@gmail.com (C.-L.P.) \\ * Correspondence: wjchang@mail.ntou.edu.tw; Tel.: +886-2-2462-2192 (ext. 7110)
}

check for

updates

Citation: Chang, W.-J.; Tsai, M.-H. Pen, C.-L. Observer-Based Fuzzy Controller Design for Nonlinear Discrete-Time Singular Systems via Proportional Derivative Feedback Scheme. Appl. Sci. 2021, 11, 2833. https://doi.org/10.3390/app11062833

Received: 7 March 2021

Accepted: 19 March 2021

Published: 22 March 2021

Publisher's Note: MDPI stays neutral with regard to jurisdictional claims in published maps and institutional affiliations.

Copyright: (c) 2021 by the authors. Licensee MDPI, Basel, Switzerland. This article is an open access article distributed under the terms and conditions of the Creative Commons Attribution (CC BY) license (https:/ / creativecommons.org/licenses/by/ $4.0 /)$.

\begin{abstract}
This paper investigates the observer-based fuzzy controller design method for nonlinear discrete-time singular systems that are represented by Takagi-Sugeno (T-S) fuzzy models. At first, the nonlinearity can be well-approximated with several local linear input-output relationships. The parallel distributed compensation (PDC) technology and the proportional derivative (PD) feedback scheme are then employed to construct the observer-based fuzzy controller. To solve the problem of unmeasured states, the impulsive phenomenon of singular systems, and the PD scheme's reasonableness, a novel observer-based fuzzy controller is developed. By using the Lyapunov theory and projection lemma, the stability criteria are built in terms of linear matrix inequalities (LMI). Moreover, the gains of fuzzy controller and fuzzy observer can be calculated synchronously by using convex optimization algorithms. Finally, a biological economic system is provided to verify the effectiveness of the proposed fuzzy control method.
\end{abstract}

Keywords: nonlinear singular systems; T-S fuzzy model; observer design; proportional derivative control

\section{Introduction}

Singular systems, also called descriptor systems or implicit systems, are important in the control field since their general representation. Lots of practical systems can be described as singular state-space models [1-6]. For instance, DC motor systems, bioeconomic models, electrical circuit systems, and network control systems. Before studying the stabilization issues for discrete-time singular systems, regularity and causality need to be discussed. Because of the algebraic equations for the singular models, there may exist more than one solution. It results in the unwanted impulse terms that emerged in state trajectories to destroy singular systems' stability. In the past few decades, the above properties are discussed with matrix decompositions [7,8]. Based on the matrix decomposition techniques, some sufficient definitions concerning regular, causal, and admissible were presented [9]. Then, numerous related control issues were investigated in [9-12], e.g., the event-triggered control, sliding mode control, output feedback control, and robust $H_{\infty}$ control, etc.

On the other hand, nonlinearity always exists in practical systems. To discuss the stabilization issue of nonlinear systems, the T-S fuzzy modeling approach was first proposed in [13]. Compared to the Mamdani fuzzy models, the calculations of fuzzy controller design and the systems' structure of the T-S fuzzy models are relatively complex. However, the stability analysis of T-S fuzzy models is more systematic with a mathematical foundation, i.e., it possesses well mathematical basis and structure extension. Thus, the T-S fuzzy models attracted numerous researchers' attention in the past two decades. The T-S fuzzy modeling approach is a conceptually simple and effective method to approximate nonlinear behaviors. Via reasonable fuzzy sets and fuzzy inferences, several linear subsystems are constructed by a series of IF-THEN fuzzy rules. Then, the final output of the T-S 
systems can be obtained with membership functions. Some practical applications of the T-S fuzzy models, such as temperature control [14] and prediction of generated electricity [15], have been investigated to address their advantages. For stabilizing the T-S fuzzy systems, PDC technology [16] was applied to construct fuzzy control schemes. This idea is that for each local subsystem, the controller can be designed with linear control techniques. Some normal systems, such as the ship steering system [17], ship fin stabilizing system [18], and drum boiler system [19], have been discussed. This modeling approach and controller design technique are not only applicable for normal systems but also for singular systems. Several stabilization issues of T-S fuzzy singular systems were researched by PDC-based fuzzy controllers [20-22].

In the past decade, the state feedback controller was constructed to stabilize the T-S fuzzy singular systems. To overcome the problem that the system's states are not always measurable, some control schemes have been investigated, for example, static output feedback [11], observer-based feedback [23], and dynamic output feedback [24]. However, if only using a traditional state feedback controller to singular systems, the complex model transformations for system matrices are unavoidable. To simplify the derivation process of the stability criteria, the PD feedback scheme was used to discuss control problems in [25-32]. For instance in [25] the stabilization issues for continuous-time and discretetime linear switched singular systems were studied via PD control strategy with the free-weighting matrix technique. In [26], the passive $H_{\infty}$ control for the discrete-time linear switched singular systems was studied by using the Schur lemma. The related PD control problems were studied in [27-32]. The PD observer issue for discrete-time singular systems was studied in [33]. In [25,26], the stabilization via PD state feedback fuzzy control scheme was studied. Referring to Remark 7 of [25], the PD controller can be reasonably used for discrete-time cases in the case of a high accuracy state observer. However, the observerbased fuzzy control was not developed in $[25,26]$ due to the computation complexity. The observer-based PD fuzzy control scheme is still an open problem for the discrete-time nonlinear systems to the best of the authors' knowledge. Thus, the motivation of this paper is to overcome the above difficulty and develop a reasonable observer-based PD feedback fuzzy control scheme for discrete-time nonlinear singular systems.

For the advantages of T-S fuzzy models and the motivations mentioned above, the stabilization problem of observer-based control for discrete-time T-S fuzzy singular systems was studied in this paper. By using the T-S fuzzy modeling approach, the nonlinear singular systems can be approximated efficiently with some local linear models blending by membership functions. Meanwhile, the observer-based fuzzy controller was designed to reasonably use the PD feedback scheme in discrete-time cases. Then, according to Lyapunov theory and the transformation technologies $[34,35]$, the inverse problems were solved and sufficient stability criteria can be developed in terms of LMI, which can be conveniently calculated with convex optimization algorithms [36]. The main contributions of this paper can be summarized as follows: (i) The proposed observer feedback fuzzy control method is more generalized to conform to reality than the state feedback fuzzy control approaches $[25,26]$ when the system states cannot be measured. (ii) Extra freedom variables of the LMI stability conditions derived by projection lemma let designers get more degrees of freedom when solving the observer-based fuzzy control problems. (iii) With the fuzzy observer design and PD feedback scheme, an observer-based PD feedback fuzzy controller with novel stability conditions was derived to ensure the stability of discrete-time T-S fuzzy singular systems. To verify the effectiveness and application of the proposed observer-based fuzzy control method, the control problem of a bio-economic system was presented. Some comparisons between the proposed observer-based fuzzy control method and previous fuzzy control approaches were also provided in the simulations.

The organization of this paper is presented as follows. The nonlinear singular system is represented as a T-S fuzzy model, and some necessary assumptions are proposed in Section 2. The sufficient stability conditions for the singular system are derived in Section 3. A biological economic system is proposed to verify the effectiveness of the 
proposed fuzzy control method in Section 4. Finally, the conclusions and future works are collected in Section 5.

\section{System Descriptions and Problem Statements}

To represent the nonlinear singular systems, the T-S fuzzy modeling approach was applied in this paper. The T-S fuzzy singular system can be represented as follows:

Plant Rule $i$ :

IF $\Im_{1}(k)$ is $\mathrm{M}_{i 1}$ and $\ldots$ and $\Im_{q}(k)$ is $\mathrm{M}_{i q}$ THEN

$$
\begin{gathered}
\mathbf{E} x(k+1)=\mathbf{A}_{i} x(k)+\mathbf{B}_{i} u(k) \\
y(k)=\mathbf{C}_{i} x(k)
\end{gathered}
$$

where $\Im(k)=\left[\begin{array}{llll}\Im_{1}(k) & \Im_{2}(k) & \ldots & \Im_{q}(k)\end{array}\right], i=1, \ldots, n$ and $n$ is the number of rules, $\mathrm{M}_{i q}$ are fuzzy sets, $q$ is the number of premise variables, $x(k) \in \Re^{m_{x}}, u(k) \in \Re^{m_{u}}$ and $y(k) \in \Re^{m_{y}}$ are the vector of state, control input, and output, respectively. $\mathbf{A}_{i}, \mathbf{B}_{i}$ and $\mathbf{C}_{i}$ are constant matrices with appropriate dimensions. $\mathbf{E}$ is a matrix with $\operatorname{rank}(\mathbf{E})<m_{x}$. By blending all the fuzzy rules, one can infer the T-S fuzzy singular system as follows:

$$
\begin{gathered}
\mathbf{E} x(k+1)=\sum_{i=1}^{n} h_{i}(\Im(k))\left\{\mathbf{A}_{i} x(k)+\mathbf{B}_{i} u(k)\right\} \\
y(k)=\sum_{i=1}^{n} h_{i}(\Im(k))\left\{\mathbf{C}_{i} x(k)\right\}
\end{gathered}
$$

where $h_{i}(\Im(k))=\prod_{l=1}^{q} \mathrm{M}_{i l}\left(\Im_{l}(k)\right) / \sum_{i=1}^{n} \prod_{l=1}^{q} \mathrm{M}_{i l}\left(\Im_{l}(k)\right), h_{i}(\Im(k)) \geq 0$ and $\sum_{i=1}^{n} h_{i}(\Im(k))=1$. Due to the limitation of space, the abbreviation $h_{i}(\Im(t)) \triangleq h_{i}$ is defined in the following context.

Considering the negative effects caused by algebraic equations, regularity and causality are the premise criteria and need to be discussed via model transformations $[7,8]$. In recent years, the PD feedback scheme is applied for singular systems to eliminate the impulse terms $[25,26]$. Because singular systems are transformed into normal state-space models, the above properties can be naturally satisfied. Referring to [25], however, in order to reasonably use the PD control method for discrete-time singular systems, the observer should be constructed to estimate system's states. As same as $[25,26]$, it is assumed that the current time step is instant $k+1$ and the previous time step is instant $k$. For online implementation, it is expected that the time taken to estimate the state $x(k+1)$ is smaller compared with the sampling period. The T-S fuzzy PD observer can be constructed as follows:

Observer Rule $i$ :

IF $\Im_{1}(k)$ is $\mathrm{M}_{i 1}$ and $\ldots$ and $\Im_{q}(k)$ is $\mathrm{M}_{i q}$ THEN

$$
\mathbf{E} \hat{x}(k+1)=\mathbf{A}_{i} \hat{x}(k)+\mathbf{B}_{i} u(k)+\mathbf{L}_{p i}\left(y(k)-\mathbf{C}_{j} \hat{x}(k)\right)+\mathbf{L}_{d i}\left(y(k+1)-\mathbf{C}_{j} \hat{x}(k+1)\right)
$$

where $\hat{x}(k) \in \mathbb{R}^{m_{x}}$ is the estimated state vector, $\mathbf{L}_{p i}$ and $\mathbf{L}_{d i}$ are the observer gains with appropriate dimensions.

The overall T-S fuzzy PD observer as follows:

$$
\mathbf{E} \hat{x}(k+1)=\sum_{i=1}^{n} \sum_{j=1}^{n} h_{i} h_{j}\left\{\mathbf{A}_{i} \hat{x}(k)+\mathbf{B}_{i} u(k)+\mathbf{L}_{p i}\left(y(k)-\mathbf{C}_{j} \hat{x}(k)\right)+\mathbf{L}_{d i}\left(y(k+1)-\mathbf{C}_{j} \hat{x}(k+1)\right)\right\}
$$

With output observations, the system states can be estimated via PD fuzzy observer. Next, to ensure the stability of the system (3), the observer-based PD controller is represented as follows:

Controller Rule $i$ : 
IF $\Im_{1}(k)$ is $\mathrm{M}_{i 1}$ and $\ldots$ and $\Im_{q}(k)$ is $\mathrm{M}_{i q}$ THEN

$$
u(k)=-\mathbf{F}_{p i} \hat{x}(k)-\mathbf{F}_{d i} \hat{x}(k+1)
$$

where $\mathbf{F}_{p i}$ and $\mathbf{F}_{d i}$ are the controller gains. The final control output is constructed as

$$
u(k)=\sum_{i=1}^{n} h_{i}\left\{-\mathbf{F}_{p i} \hat{x}(k)-\mathbf{F}_{d i} \hat{x}(k+1)\right\}
$$

Subsequently, the closed-loop T-S fuzzy singular system is obtained by substituting the observer-based PD fuzzy controller (8) into the system (3). The overall closed-loop T-S fuzzy singular system is represented as follows:

$$
\mathbf{E} x(k+1)=\sum_{i=1}^{n} \sum_{j=1}^{n} h_{i} h_{j}\left\{\mathbf{A}_{i} x(k)-\mathbf{B}_{i} \mathbf{F}_{p j} \hat{x}(k)-\mathbf{B}_{i} \mathbf{F}_{d j} \hat{x}(k+1)\right\}
$$

According to motivations as mentioned above, the observer-based control scheme is built in this work. One of the purposes of this paper is to estimate the systems' states and to ensure the stability of the system (9). As the estimation errors converge to zero, the estimated states can replace the actual states. Next, define the estimation error as $e(k)=x(k)-\hat{x}(k)$, the following equation can be inferred from (9).

$$
\begin{aligned}
& \mathbf{E} x(k+1)=\sum_{i=1}^{n} \sum_{j=1}^{n} h_{i} h_{j}\left\{\mathbf{A}_{i} x(k)-\mathbf{B}_{i} \mathbf{F}_{p j}(x(k)-e(k))-\mathbf{B}_{i} \mathbf{F}_{d j}(x(k+1)-e(k+1))\right\} \\
& =\sum_{i=1}^{n} \sum_{j=1}^{n} h_{i} h_{j}\left\{\mathbf{A}_{i} x(k)-\mathbf{B}_{i} \mathbf{F}_{p j} x(k)-\mathbf{B}_{i} \mathbf{F}_{d j} x(k+1)+\mathbf{B}_{i} \mathbf{F}_{p j} e(k)+\mathbf{B}_{i} \mathbf{F}_{d j} e(k+1)\right\}
\end{aligned}
$$

The closed-loop T-S fuzzy singular system is presented as aforementioned. Subsequently, the errors systems have to be built to guarantee estimation errors converge to zero as $t \rightarrow \infty$. Now multiplying the matrix $\mathbf{E}$ on the left side of the difference function $e(k+1)=x(k+1)-\hat{x}(k+1)$, one can obtain the following closed-loop error systems.

$$
\mathbf{E} e(k+1)=\mathbf{E} x(k+1)-\mathbf{E} \hat{x}(k+1)
$$

$$
\begin{gathered}
=\sum_{i=1}^{n} \sum_{j=1}^{n} h_{i} h_{j}\left\{\mathbf{A}_{i} x(k)+\mathbf{B}_{i} u(k)-\left(\mathbf{A}_{i} \hat{x}(k)+\mathbf{B}_{i} u(k)+\mathbf{L}_{p i}\left(\mathbf{C}_{j} x(k)-\mathbf{C}_{j} \hat{x}(k)\right)+\mathbf{L}_{d i}\left(\mathbf{C}_{j} x(k+1)-\mathbf{C}_{j} \hat{x}(k+1)\right)\right)\right\} \\
=\sum_{i=1}^{n} \sum_{j=1}^{n} h_{i} h_{j}\left\{\left(\mathbf{A}_{i}-\mathbf{L}_{p i} \mathbf{C}_{j}\right) e(k)-\mathbf{L}_{d i} \mathbf{C}_{j} e(k+1)\right\}
\end{gathered}
$$

The following augmented systems can be further obtained from the system (10) and (11).

$$
\mathbf{E}_{R i j}(h) \widetilde{x}(k+1)=\mathbf{A}_{R i j}(h) \widetilde{x}(k)
$$

where $\widetilde{x}(k)=\left[\begin{array}{ll}x^{\mathrm{T}}(k) & e^{\mathrm{T}}(k)\end{array}\right]^{\mathrm{T}}, \mathbf{E}_{R i j}(h)=\sum_{i=1}^{n} \sum_{j=1}^{n} h_{i} h_{j} \mathbf{E}_{R i j}, \mathbf{A}_{R i j}(h)=\sum_{i=1}^{n} \sum_{j=1}^{n} h_{i} h_{j} \mathbf{A}_{R i j}$, $\mathbf{E}_{R i j}=\left[\begin{array}{cc}\mathbf{E}+\mathbf{B}_{i} \mathbf{F}_{d j} & -\mathbf{B}_{i} \mathbf{F}_{d j} \\ 0 & \mathbf{E}+\mathbf{L}_{d i} \mathbf{C}_{j}\end{array}\right]$ and $\mathbf{A}_{R i j}=\left[\begin{array}{cc}\mathbf{A}_{i}-\mathbf{B}_{i} \mathbf{F}_{p j} & \mathbf{B}_{i} \mathbf{F}_{p j} \\ 0 & \mathbf{A}_{i}-\mathbf{L}_{p i} \mathbf{C}_{j}\end{array}\right]$.

Furthermore, the following assumptions concerning controllability and observability of T-S fuzzy singular systems are proposed. Similar assumptions can be found in [26,33]. In addition, the lemmas are presented to convert stability conditions into LMI form.

Assumption 1. System (3) is completely controllable if the following conditions are satisfied.

$$
\operatorname{rank}\left[s \mathbf{E}-\mathbf{A}_{i} \quad \mathbf{B}_{i}\right]=m_{x} \text { and } \operatorname{rank}\left[\begin{array}{cc}
\mathbf{E} & \mathbf{B}_{i}
\end{array}\right]=m_{x}, \forall s \in \mathbb{C}_{+} .
$$


where $\mathbb{C}_{+}$is the open right-half of the complex plane.

Assumption 2. The system (3) is completely observable if the following conditions are held.

$$
\operatorname{rank}\left[\begin{array}{c}
s \mathbf{E}-\mathbf{A}_{i} \\
\mathbf{C}_{i}
\end{array}\right]=m_{x} \text { and } \operatorname{rank}\left[\begin{array}{c}
\mathbf{E} \\
\mathbf{C}_{i}
\end{array}\right]=m_{x}, \forall s \in \mathbb{C}_{+} .
$$

Remark 1. Under the necessary assumptions as mentioned previously, one knows that the matrix $\mathbf{E}_{R i j}$ is a full rank matrix. (i.e., $\mathbf{E}_{R i j}$ is invertible.) The system (10) is equivalent to the following representation.

$$
\widetilde{x}(k+1)=\mathbf{E}_{R i j}^{-1}(h) \mathbf{A}_{R i j}(h) \widetilde{x}(k)
$$

Obviously, one can find the augmented system (13) is a normal state-space model since the controller gain $\mathbf{F}_{d j}$ and the observer gain $\mathbf{L}_{d i}$, i.e., the regularity and causality of the system (3) are held.

Lemma 1. [34] (Projection Lemma) Given matrices $\boldsymbol{\Omega}, \boldsymbol{\Lambda}$ and symmetric matrix $\boldsymbol{\Phi}$ which satisfies $\operatorname{rank}(\boldsymbol{\Omega})<\operatorname{rank}(\boldsymbol{\Phi})$ and $\operatorname{rank}(\boldsymbol{\Lambda})<\operatorname{rank}(\boldsymbol{\Phi})$, if and only if there exists arbitrary matrix $\Pi$ such that

$$
\boldsymbol{\Phi}+\boldsymbol{\Omega}^{\mathrm{T}} \boldsymbol{\Pi} \boldsymbol{\Lambda}+\boldsymbol{\Lambda}^{\mathrm{T}} \boldsymbol{\Pi}^{\mathrm{T}} \boldsymbol{\Omega}<0
$$

and the following inequalities are held simultaneously.

$$
\boldsymbol{\Omega}_{\perp}^{\mathrm{T}} \boldsymbol{\Phi} \boldsymbol{\Omega}_{\perp}<0 \text { and } \boldsymbol{\Lambda}_{\perp}^{\mathrm{T}} \boldsymbol{\Phi} \boldsymbol{\Lambda}_{\perp}<0
$$

where $\bullet \perp$ denotes the null-space matrix of $\bullet$.

Lemma 2. [35] A scalar $\alpha>0$ exists to satisfy the following inequality if matrices $\mathbf{U}$ and $\mathbf{\Xi}<0$ with appropriate dimension such that $\mathbf{U}^{\mathrm{T}} \boldsymbol{\Xi} \mathbf{U} \leq 0$ is held.

$$
\mathbf{U}^{\mathrm{T}} \boldsymbol{\Xi} \mathbf{U} \leq-\alpha\left(\mathbf{U}^{\mathrm{T}}+\mathbf{U}\right)-\alpha^{2} \boldsymbol{\Xi}^{-1}
$$

In the next section, the stabilization issue of the system (13) is proposed by using the Lyapunov stability theory. Moreover, the stability conditions will be converted into LMI form through the above lemmas. Then, the convex optimization algorithms [36] can be employed to solve these LMI stability conditions.

\section{Main Results}

The main object of this paper is to discuss the stabilization issues of T-S fuzzy singular systems. Via the observer-based PD fuzzy control strategy and Lyapunov stability theory, some sufficient stability conditions for the system (13) are derived as follows:

Theorem 1. If there exist matrices $\mathbf{P}_{1}=\mathbf{P}_{1}^{\mathrm{T}}>0, \mathbf{P}_{2}=\mathbf{P}_{2}^{\mathrm{T}}>0, \mathbf{F}_{p j}, \mathbf{F}_{d j}, \mathbf{L}_{p i}, \mathbf{L}_{d i}, \mathbf{Z}_{1}$ and $\mathbf{Z}_{2}$ such that the following condition is held, the augmented system (13) is stable.

$$
\left[\begin{array}{cc}
\widetilde{\mathbf{P}}-H e\left\{\boldsymbol{\Pi}^{\mathrm{T}} \mathbf{E}_{R i j}\right\} & \boldsymbol{\Pi}^{\mathrm{T}} \mathbf{A}_{R i j} \\
* & -\widetilde{\mathbf{P}}
\end{array}\right]<0
$$

where $\widetilde{\mathbf{P}}=\left[\begin{array}{cc}\mathbf{P}_{1} & 0 \\ 0 & \mathbf{P}_{2}\end{array}\right], \boldsymbol{\Pi}=\left[\begin{array}{cc}\mathbf{Z}_{1}^{-1} & 0 \\ 0 & \mathbf{Z}_{2}\end{array}\right]$ and He$\{\bullet\}$ denotes the shorthand of $\bullet+\bullet \bullet^{\mathrm{T}}$. The $\mathbf{E}_{\text {Rij }}$ and $\mathbf{A}_{\text {Rij }}$ are pre-defined in (12). 
Proof. Choosing the Lyapunov quadratic function $V(\widetilde{x}(k))=\widetilde{x}^{T}(k) \widetilde{\mathbf{P}} \widetilde{x}(k)$, one can obtain the first forward difference of $V(\widetilde{x}(k))$ as following equation.

$$
\begin{gathered}
\Delta V(\widetilde{x}(k))=\widetilde{x}^{\mathrm{T}}(k+1) \widetilde{\mathbf{P}} \widetilde{x}(k+1)-\widetilde{x}^{\mathrm{T}}(k) \widetilde{\mathbf{P}} \widetilde{x}(k) \\
=\widetilde{x}^{\mathrm{T}}(k)\left(\mathbf{E}_{R i j}^{-1}(h) \mathbf{A}_{R i j}(h)\right)^{\mathrm{T}} \widetilde{\mathbf{P}}\left(\mathbf{E}_{R i j}^{-1}(h) \mathbf{A}_{R i j}(h)\right) \widetilde{x}(k)-\widetilde{x}^{\mathrm{T}}(k) \widetilde{\mathbf{P}} \widetilde{x}(k) \\
=\widetilde{x}^{\mathrm{T}}(k)\left(\mathbf{A}_{R i j}^{\mathrm{T}}(h) \mathbf{E}_{R i j}^{-\mathrm{T}}(h) \widetilde{\mathbf{P}} \mathbf{E}_{R i j}^{-1}(h) \mathbf{A}_{R i j}(h)-\widetilde{\mathbf{P}}\right) \widetilde{x}(k)
\end{gathered}
$$

Based on $\sum_{i=1}^{n} h_{i}=1$ and $0 \leq h_{i} \leq 1$, the condition (17) can be rewritten as follows:

$$
\left[\begin{array}{cc}
\widetilde{\mathbf{P}}-H e\left\{\boldsymbol{\Pi}^{\mathrm{T}} \mathbf{E}_{R i j}(h)\right\} & \boldsymbol{\Pi}^{\mathrm{T}} \mathbf{A}_{R i j}(h) \\
* & -\widetilde{\mathbf{P}}
\end{array}\right]=\boldsymbol{\Xi}+H e\left\{\mathbf{\Psi}_{i j}^{\mathrm{T}} \boldsymbol{\Pi} \boldsymbol{\Lambda}\right\}<0
$$

where $\mathbf{\Psi}_{i j}=\left[\begin{array}{ll}-\mathbf{E}_{R i j}(h) & \mathbf{A}_{R i j}(h)\end{array}\right], \boldsymbol{\Lambda}=\left[\begin{array}{ll}\mathbf{I} & 0\end{array}\right], \boldsymbol{\Xi}=\left[\begin{array}{cc}\widetilde{\mathbf{P}} & 0 \\ 0 & -\widetilde{\mathbf{P}}\end{array}\right], \mathbf{I}$ is identify matrix and ${ }^{*}$ denotes the transposed elements of the symmetric position.

According to Lemma 1, the following inequalities can be found from (19).

$$
\mathbf{A}_{R i j}^{\mathrm{T}}(h) \mathbf{E}_{R i j}^{-\mathrm{T}}(h) \widetilde{\mathbf{P}} \mathbf{E}_{R i j}^{-1}(h) \mathbf{A}_{R i j}(h)-\widetilde{\mathbf{P}}=\mathbf{\Psi}_{i j \perp}^{\mathrm{T}} \boldsymbol{\Xi} \mathbf{\Psi}_{i j \perp}<0
$$

and

$$
-\widetilde{\mathbf{P}}=\boldsymbol{\Lambda}_{\perp}^{\mathrm{T}} \boldsymbol{\Xi} \boldsymbol{\Lambda}_{\perp}<0
$$

where $\mathbf{\Psi}_{i j \perp}^{\mathrm{T}}=\left[\begin{array}{ll}\mathbf{A}_{R i j}^{\mathrm{T}}(h) \mathbf{E}_{R i j}^{-\mathrm{T}}(h) & \mathbf{I}\end{array}\right]$ and $\boldsymbol{\Lambda}_{\perp}^{\mathrm{T}}=\left[\begin{array}{ll}0 & \mathbf{I}\end{array}\right]$. Clearly, the $\Delta V(\widetilde{x}(k))<0$ if $\Psi_{i j \perp}^{\mathrm{T}} \Xi \boldsymbol{\Psi}_{i j \perp}<0$. Based on Lyapunov theory, it further means that the systems (13) is stable. The proof of Theorem 1 is completed.

Remark 2. According to [32], a similar Lyapunov function is chosen to derive the stability conditions for the controlled systems. However, some conservatisms exist due to the use of diagonal matrices of the Lyapunov matrix and the freedom variables obtained by projection lemma, i.e., choosing a less conservative Lyapunov function and suitable variables of projection lemma is still a challenging problem for developing stability criteria.

Please note that the stability conditions (17) are not LMI forms. To conveniently solve the proposed stability problem by using convex optimization algorithms [36], it is necessary to transform the conditions of Theorem 1 to LMI forms. The complete transformations were given in the following theorem.

Theorem 2. The augmented system (13) is stable if there exist matrices $\mathbf{X}>0, \mathbf{P}_{2}>0, \mathbf{Y}_{p j}, \mathbf{Y}_{d j}$, $\mathbf{K}_{p i}, \mathbf{K}_{d i}, \mathbf{Z}_{1}, \mathbf{Z}_{2}$ and a scalar $\alpha>0$ such that the following conditions are held.

$$
\left[\begin{array}{ccccccc}
\boldsymbol{\Theta}_{1 i i}+\mathbf{X} & \mathbf{B}_{i} \mathbf{Y}_{d i} & \boldsymbol{\Theta}_{2 i i} & \mathbf{B}_{i} \mathbf{Y}_{p i} & 0 & 0 & 0 \\
* & -H e\left\{\alpha \mathbf{Z}_{1}\right\} & 0 & 0 & \alpha \mathbf{I} & 0 & 0 \\
* & * & -2 \alpha \mathbf{I} & 0 & 0 & \alpha \mathbf{I} & 0 \\
* & * & * & -H e\left\{\alpha \mathbf{Z}_{1}\right\} & 0 & 0 & \alpha \mathbf{I} \\
* & * & * & * & \boldsymbol{\Theta}_{3 i i}+\mathbf{P}_{2} & 0 & \boldsymbol{\Theta}_{4 i i} \\
* & * & * & * & * & -\mathbf{X} & 0 \\
* & * & * & * & * & * & -\mathbf{P}_{2}
\end{array}\right]<0
$$




$$
\begin{aligned}
& {\left[\begin{array}{ccccccc}
\mathbf{X}+\boldsymbol{\Theta}_{1 i j} & \frac{\mathbf{B}_{i} \mathbf{Y}_{d j}+\mathbf{B}_{j} \mathbf{Y}_{d i}}{2} & \boldsymbol{\Theta}_{2 i j} & \frac{\mathbf{B}_{i} \mathbf{Y}_{p j}+\mathbf{B}_{j} \mathbf{Y}_{p i}}{2} & 0 & 0 & 0 \\
* & -H e\left\{\alpha \mathbf{Z}_{1}\right\} & 0 & 0 & \alpha \mathbf{I} & 0 & 0 \\
* & * & -2 \alpha \mathbf{I} & 0 & 0 & \alpha \mathbf{I} & 0 \\
* & * & * & -H e\left\{\alpha \mathbf{Z}_{1}\right\} & 0 & 0 & \alpha \mathbf{I} \\
* & * & * & * & \mathbf{P}_{2}+\mathbf{\Theta}_{3 i j} & 0 & \boldsymbol{\Theta}_{4 i j} \\
* & * & * & * & * & -\mathbf{X} & 0 \\
* & * & * & * & * & * & -\mathbf{P}_{2}
\end{array}\right]<0} \\
& \text { where } \mathbf{\Theta}_{1 i i}=-H e\left\{\mathbf{E Z}_{1}+\mathbf{B}_{i} \mathbf{Y}_{d i}\right\}, \mathbf{\Theta}_{1 i j}=-H e\left\{\mathbf{E Z}_{1}+\frac{\mathbf{B}_{i} \mathbf{Y}_{d j}+\mathbf{B}_{j} \mathbf{Y}_{d i}}{2}\right\}, \boldsymbol{\Theta}_{2 i i}=\mathbf{A}_{i} \mathbf{Z}_{1}-\mathbf{B}_{i} \mathbf{Y}_{p i} \text {, } \\
& \boldsymbol{\Theta}_{2 i j}=\frac{\mathbf{A}_{i} \mathbf{Z}_{1}-\mathbf{B}_{i} \mathbf{Y}_{p j}+\mathbf{A}_{j} \mathbf{Z}_{1}-\mathbf{B}_{j} \mathbf{Y}_{p i}}{2}, \boldsymbol{\Theta}_{3 i i}=-H e\left\{\mathbf{Z}_{2}^{\mathrm{T}} \mathbf{E}+\mathbf{K}_{d i} \mathbf{C}_{i}\right\}, \boldsymbol{\Theta}_{3 i j}= \\
& -H e\left\{\mathbf{Z}_{2}^{\mathrm{T}} \mathbf{E}+\frac{\mathbf{K}_{d i} \mathbf{C}_{j}+\mathbf{K}_{d j} \mathbf{C}_{i}}{2}\right\}, \boldsymbol{\Theta}_{4 i i}=\mathbf{Z}_{2}^{\mathrm{T}} \mathbf{A}_{i}-\mathbf{K}_{p i} \mathbf{C}_{i} \text { and } \mathbf{\Theta}_{4 i j}=\frac{\mathbf{Z}_{2}^{\mathrm{T}} \mathbf{A}_{i}-\mathbf{K}_{p i} \mathbf{C}_{j}+\mathbf{Z}_{2}^{\mathrm{T}} \mathbf{A}_{j}-\mathbf{K}_{p j} \mathbf{C}_{i}}{2} \text {. }
\end{aligned}
$$

Proof. The following inequality is an equivalent form of the stability condition (17).

$$
\left[\begin{array}{cccc}
\mathbf{P}_{1}-H e\left\{\mathbf{Z}_{1}^{-\mathrm{T}}\left(\mathbf{E}+\mathbf{B}_{i} \mathbf{F}_{d j}\right)\right\} & \mathbf{Z}_{1}^{-\mathrm{T}} \mathbf{B}_{i} \mathbf{F}_{d j} & \mathbf{Z}_{1}^{-\mathrm{T}}\left(\mathbf{A}_{i}-\mathbf{B}_{i} \mathbf{F}_{p j}\right) & \mathbf{Z}_{1}^{-\mathrm{T}} \mathbf{B}_{i} \mathbf{F}_{p j} \\
* & \mathbf{P}_{2}-H e\left\{\mathbf{Z}_{2}^{\mathrm{T}} \mathbf{E}+\mathbf{Z}_{2}^{\mathrm{T}} \mathbf{L}_{d i} \mathbf{C}_{j}\right\} & 0 & \mathbf{Z}_{2}^{\mathrm{T}}\left(\mathbf{A}_{i}-\mathbf{L}_{p i} \mathbf{C}_{j}\right) \\
* & * & -\mathbf{P}_{1} & 0 \\
* & * & * & -\mathbf{P}_{2}
\end{array}\right]<0
$$

Then, pre- and post-multiplying the matrix $\widetilde{\mathbf{Z}}_{1}^{\mathrm{T}}$ and its transpose matrix on both sides of (24), one can obtain the following equation by defining $\mathbf{X}=\mathbf{Z}_{1}^{\mathrm{T}} \mathbf{P}_{1} \mathbf{Z}_{1}, \mathbf{Y}_{p j}=\mathbf{F}_{p j} \mathbf{Z}_{1}$, $\mathbf{Y}_{d j}=\mathbf{F}_{d j} \mathbf{Z}_{1}, \mathbf{K}_{p i}=\mathbf{Z}_{2}^{\mathrm{T}} \mathbf{L}_{p i}$ and $\mathbf{K}_{d i}=\mathbf{Z}_{2}^{\mathrm{T}} \mathbf{L}_{d i}$. Where $\widetilde{\mathbf{Z}}_{1}=\operatorname{diag}\left[\begin{array}{llll}\mathbf{Z}_{1} & \mathbf{Z}_{1} & \mathbf{Z}_{1} & \mathbf{Z}_{1}\end{array}\right]$.

$$
\left[\begin{array}{cccc}
\mathbf{X}-H e\left\{\mathbf{E Z}_{1}+\mathbf{B}_{i} \mathbf{Y}_{d j}\right\} & \mathbf{B}_{i} \mathbf{Y}_{d j} & \mathbf{A}_{i} \mathbf{Z}_{1}-\mathbf{B}_{i} \mathbf{Y}_{p j} & \mathbf{B}_{i} \mathbf{Y}_{p j} \\
* & \mathbf{Z}_{1}^{\mathrm{T}}\left(\mathbf{P}_{2}+\mathbf{\Theta}_{3 i j}\right) \mathbf{Z}_{1} & 0 & \mathbf{Z}_{1}^{\mathrm{T}} \mathbf{\Theta}_{4 i j} \mathbf{Z}_{1} \\
* & * & -\mathbf{X} & 0 \\
* & * & * & -\mathbf{Z}_{1}^{\mathrm{T}} \mathbf{P}_{2} \mathbf{Z}_{1}
\end{array}\right]<0
$$

Notice that the right-bottom block matrix in (25) is still not an LMI framework. Thus, the following fact is proposed via Lemma 2.

$$
\begin{aligned}
& {\left[\begin{array}{ccc}
\mathbf{Z}_{1}^{\mathrm{T}}\left(\mathbf{P}_{2}+\mathbf{\Theta}_{3 i j}\right) \mathbf{Z}_{1} & 0 & \mathbf{Z}_{1}^{\mathrm{T}} \boldsymbol{\Theta}_{4 i j} \mathbf{Z}_{1} \\
* & -\mathbf{X} & 0 \\
* & * & -\mathbf{Z}_{1}^{\mathrm{T}} \mathbf{P}_{2} \mathbf{Z}_{1}
\end{array}\right]} \\
& =\left[\begin{array}{ccc}
\mathbf{Z}_{1}^{\mathrm{T}} & 0 & 0 \\
0 & \mathbf{I} & 0 \\
0 & 0 & \mathbf{Z}_{1}^{\mathrm{T}}
\end{array}\right]\left[\begin{array}{ccc}
\mathbf{P}_{2}+\mathbf{\Theta}_{3 i j} & 0 & \boldsymbol{\Theta}_{4 i j} \\
* & -\mathbf{X} & 0 \\
* & * & -\mathbf{P}_{2}
\end{array}\right]\left[\begin{array}{ccc}
\mathbf{Z}_{1} & 0 & 0 \\
0 & \mathbf{I} & 0 \\
0 & 0 & \mathbf{Z}_{1}
\end{array}\right] \leq-\alpha H e\left\{\boldsymbol{\Gamma}_{1}\right\}-\alpha^{2} \boldsymbol{\Gamma}_{2 i j}^{-1} \\
& \text { where } \boldsymbol{\Gamma}_{1}=\left[\begin{array}{ccc}
\mathbf{Z}_{1} & 0 & 0 \\
0 & \mathbf{I} & 0 \\
0 & 0 & \mathbf{Z}_{1}
\end{array}\right] \text { and } \boldsymbol{\Gamma}_{2 i j}=\left[\begin{array}{ccc}
\mathbf{P}_{2}+\boldsymbol{\Theta}_{3 i j} & 0 & \boldsymbol{\Theta}_{4 i j} \\
* & -\mathbf{X} & 0 \\
* & * & -\mathbf{P}_{2}
\end{array}\right] \text {. }
\end{aligned}
$$

Thus, the following inequality can be inferred from the condition (25).

$$
\begin{gathered}
{\left[\begin{array}{cc}
- & - \\
\boldsymbol{\Gamma}_{1 i j} & \overline{\boldsymbol{\Gamma}}_{2 i j} \\
* & \overline{\boldsymbol{\Gamma}}_{3 i j}
\end{array}\right]<0} \\
\text { for } i=j=1,2, \ldots, n
\end{gathered}
$$




$$
\begin{aligned}
& \text { where } \overline{\boldsymbol{\Gamma}}_{1 i j}=\mathbf{X}-H e\left\{\mathbf{E Z}_{1}+\mathbf{B}_{i} \mathbf{Y}_{d j}\right\}, \overline{\boldsymbol{\Gamma}}_{2 i j}=\left[\begin{array}{lll}
\mathbf{B}_{i} \mathbf{Y}_{d j} & \mathbf{A}_{i} \mathbf{Z}_{1}-\mathbf{B}_{i} \mathbf{Y}_{p j} & \mathbf{B}_{i} \mathbf{Y}_{p j}
\end{array}\right] \text { and } \\
& - \\
& \overline{\boldsymbol{\Gamma}}_{3 i j}=-\alpha H e\left\{\boldsymbol{\Gamma}_{1}\right\}-\alpha^{2} \boldsymbol{\Gamma}_{2 i j}^{-1} .
\end{aligned}
$$

By applying the Schur complement to (27), one can obtain the following condition.

$$
\begin{gathered}
{\left[\begin{array}{ccc}
- & - & \\
\boldsymbol{\Gamma}_{1 i j} & \boldsymbol{\Gamma}_{2 i j} & 0 \\
* & -\alpha H e\left\{\boldsymbol{\Gamma}_{1}\right\} & \alpha \mathbf{I} \\
* & * & \boldsymbol{\Gamma}_{2 i j}
\end{array}\right]<0} \\
\quad \text { for } i=j=1,2, \ldots, n
\end{gathered}
$$

Obviously, the convex problem (28) also can be represented as stability conditions (22) and (23) of Theorem 2. The controller gains $\mathbf{F}_{p j}=\mathbf{Y}_{p j} \mathbf{Z}_{1}^{-1}, \mathbf{F}_{d j}=\mathbf{Y}_{d j} \mathbf{Z}_{1}^{-1}$, the observer gains $\mathbf{L}_{p i}=\mathbf{Z}_{2}^{-\mathrm{T}} \mathbf{K}_{p i}, \mathbf{L}_{d i}=\mathbf{Z}_{2}^{-\mathrm{T}} \mathbf{K}_{d i}$ and the positive definite matrices $\mathbf{P}_{1}=\mathbf{Z}_{1}^{-\mathrm{T}} \mathbf{X} \mathbf{Z}_{1}^{-1}, \mathbf{P}_{2}$ can be calculated efficiently by using the convex optimization algorithms. The proof of Theorem 2 is completed.

To verify the effectiveness and applicability of the proposed fuzzy control design method, the control problem for a biological economic system is presented in the next section. Besides, some comparisons between the proposed fuzzy control method and a previous control design method are also provided in the next section to verify the advantages of the proposed observer-based PD feedback fuzzy control method.

\section{A Numerical Example}

In this section, a bio-economic system is presented as follows to verify the applicability of the proposed fuzzy control method.

$$
\begin{gathered}
\frac{d x_{1}(t)}{d t}=a x_{2}(t)-b x_{1}(t) \\
\frac{d x_{2}(t)}{d t}=\delta x_{1}(t)-b \gamma x_{2}^{2}(t)-x_{2}(t) x_{3}(t) \\
0=x_{3}(t)\left(p x_{2}(t)-c\right)-m
\end{gathered}
$$

where $x(k)=\left[\begin{array}{lll}x_{1}(k) & x_{2}(k) & x_{3}(k)\end{array}\right]^{\mathrm{T}}, x_{1}(k)$ is the population density of immature species, $x_{2}(k)$ is the population density of mature species, $x_{3}(k)$ is harvest effort of harvested mature population, and the description of other coefficients can be found in [11].

To plan the harvest strategy and maintain the sustainable development of the system, it is necessary to take action to stabilize the biological population. Let $a=0.2, b=0.7$, $\delta=0.05, \gamma=0.1, p=1, c=30, m=0$ and assume $\left|x_{2}(k)\right| \leq \mu=5$, where $\mu$ is the carrying capacity of the population, the discretized T-S fuzzy bio-economic system is purposed in [11] with the sampling period $T_{e}=0.05 \mathrm{~s}$.

$$
\begin{gathered}
\mathbf{E} x(k+1)=\sum_{i=1}^{2} h_{i}(x(k))\left\{\mathbf{A}_{i} x(k)+\mathbf{B}_{i} u(k)\right\} \\
y(k)=\sum_{i=1}^{2} h_{i}(x(k))\left\{\mathbf{C}_{i} x(k)\right\}
\end{gathered}
$$

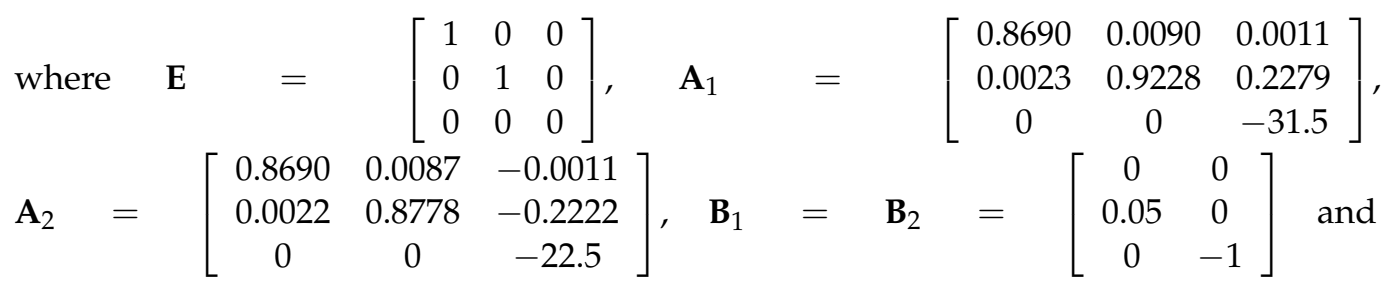


$\mathbf{C}_{1}=\mathbf{C}_{2}=\left[\begin{array}{lll}1 & 1 & 0 \\ 0 & 0 & 1\end{array}\right]$. The membership functions are $h_{1}(x(k))=\frac{1}{2}\left(1-\frac{x_{2}(k)}{\mu}\right)$, $h_{2}(x(k))=\frac{1}{2}\left(1+\frac{x_{2}(k)}{\mu}\right), \sum_{i=1}^{2} h_{i}(x(k))=1$ and stated in Figure 1.

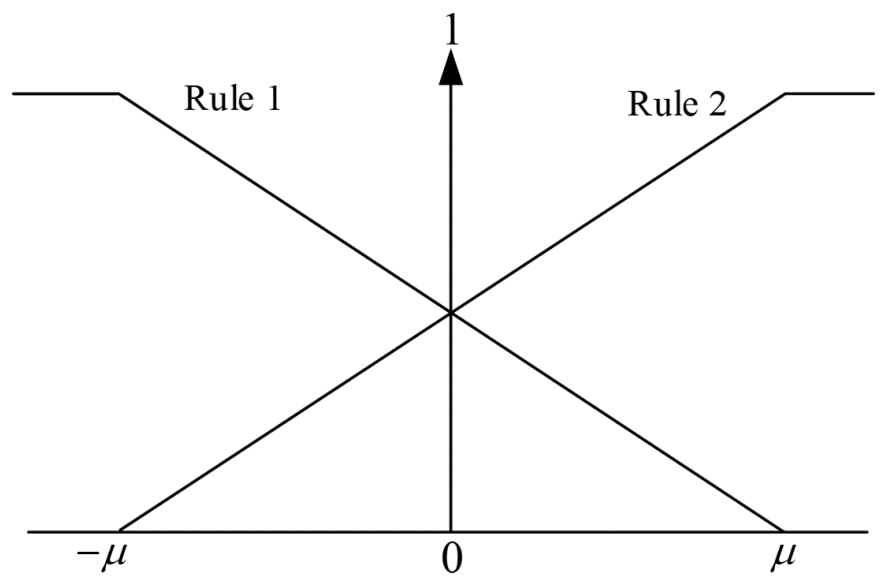

Figure 1. Membership functions $h_{i}$.

To build the observer-based fuzzy controller (8) via PD feedback scheme, the following gains can be calculated by using LMI techniques with $\alpha=20$.

$$
\begin{aligned}
& \mathbf{F}_{p 1}=\left[\begin{array}{ccc}
-0.2601 & 17.6914 & 4.2555 \\
-0.0001 & -0.0017 & 29.4621
\end{array}\right], \quad \mathbf{F}_{p 2}=\left[\begin{array}{ccc}
0.1049 & 16.4441 & -4.1554 \\
-0.0000 & -0.0014 & 21.3512
\end{array}\right], \\
& \mathbf{F}_{d 1}=\left[\begin{array}{ccc}
-0.9505 & 386.4357 & -1.2783 \\
-0.0012 & 0.0670 & -33.5144
\end{array}\right], \quad \mathbf{F}_{d 2}=\left[\begin{array}{ccc}
-0.7365 & 386.7741 & 1.9942 \\
-0.0017 & -0.0830 & -25.0700
\end{array}\right], \\
& \mathbf{L}_{p 1}^{\mathrm{T}}=\left[\begin{array}{ccc}
-0.4700 & 1.4193 & 0.0151 \\
-0.1264 & 0.3761 & -41.0435
\end{array}\right], \quad \mathbf{L}_{p 2}^{\mathrm{T}}=\left[\begin{array}{ccc}
0.8163 & 0.0429 & -0.0053 \\
0.1429 & -0.3622 & -30.4227
\end{array}\right], \\
& \mathbf{L}_{d 1}^{\mathrm{T}}=\left[\begin{array}{ccc}
567.0738 & -597.9506 & -8.3359 \\
1.1348 & -0.9925 & -138.3697
\end{array}\right] \text { and } \mathbf{L}_{d 2}^{\mathrm{T}}=\left[\begin{array}{ccc}
566.3181 & -597.1543 & -8.3570 \\
0.8390 & -0.6799 & -139.2070
\end{array}\right] \text {. }
\end{aligned}
$$
structed with a PD feedback scheme. Based on the initial condition $x(0)=\left[\begin{array}{lll}1.5 & 4 & 0\end{array}\right]^{\mathrm{T}}$ and $\widehat{x}(0)=\left[\begin{array}{lll}0 & 0 & 0\end{array}\right]^{\mathrm{T}}$, the state trajectories driven by the PD control scheme (8) are stated in Figure 2. From the simulation results, it can be found that the stability of the system (32) is achieved and the proposed fuzzy observer has been successfully employed to design a PD-based fuzzy controller. These results illustrated the proposed design method's effectiveness and superiority.

To show the advantages of the proposed design method, the following fuzzy observer and fuzzy controller are established for (33) with a previous design method developed in [23] without considering external disturbance effects.

$$
\begin{gathered}
\mathbf{E} \hat{x}(k+1)=\sum_{i=1}^{2} \sum_{i=1}^{2} h_{i}(x(k)) h_{j}(x(k))\left\{\mathbf{A}_{i} \hat{x}(k)+\mathbf{B}_{i} u(k)+\mathbf{L}_{i}\left(y(k)-\mathbf{C}_{j} \hat{x}(k)\right)\right\} \\
u(k)=-\sum_{i=1}^{2} h_{i}(x(k)) \mathbf{K}_{i} \hat{x}(k)
\end{gathered}
$$

$$
\begin{aligned}
& \text { where } \mathbf{L}_{1}^{\mathrm{T}}=\left[\begin{array}{lll}
0.4294 & 0.4524 & -0.0001 \\
0.0010 & 0.2214 & -30.9028
\end{array}\right], \mathbf{L}_{2}^{\mathrm{T}}=\left[\begin{array}{ccc}
0.4293 & 0.4305 & 0.0000 \\
-0.0010 & -0.2130 & -21.8734
\end{array}\right], \\
& \mathbf{K}_{1}=\left[\begin{array}{lll}
0.0024 & 0.9278 & 0.1699 \\
0.0001 & 0.0022 & 15.7318
\end{array}\right] \text { and } \mathbf{K}_{2}=\left[\begin{array}{ccc}
0.0023 & 0.8963 & -0.1619 \\
-0.0001 & -0.0017 & 11.1306
\end{array}\right] .
\end{aligned}
$$




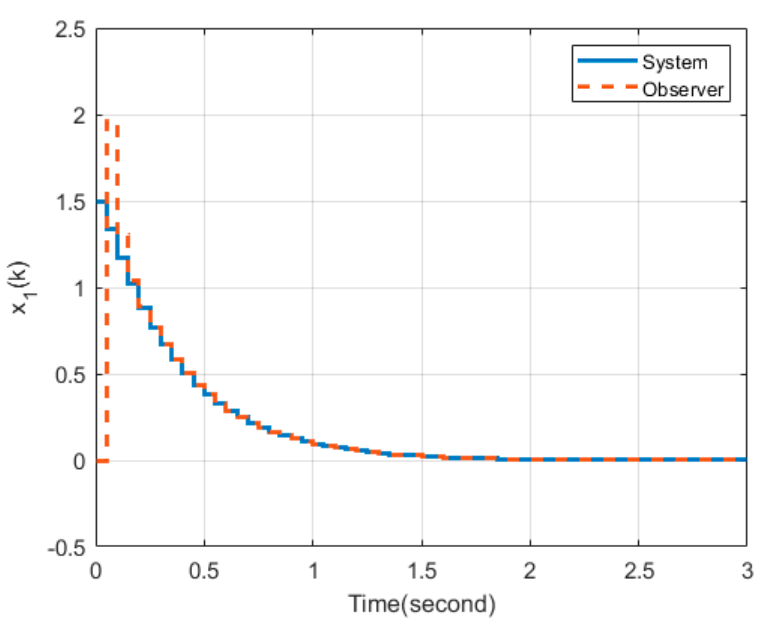

(a)

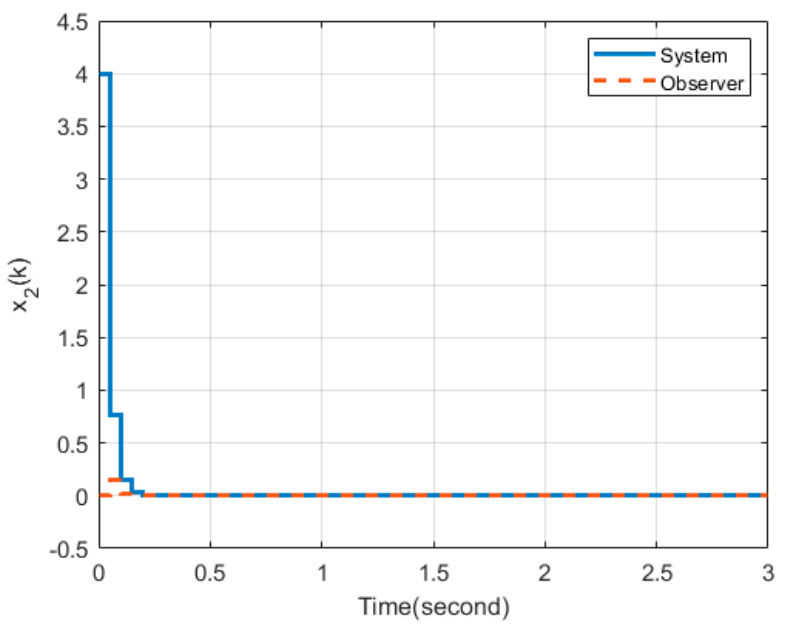

(b)

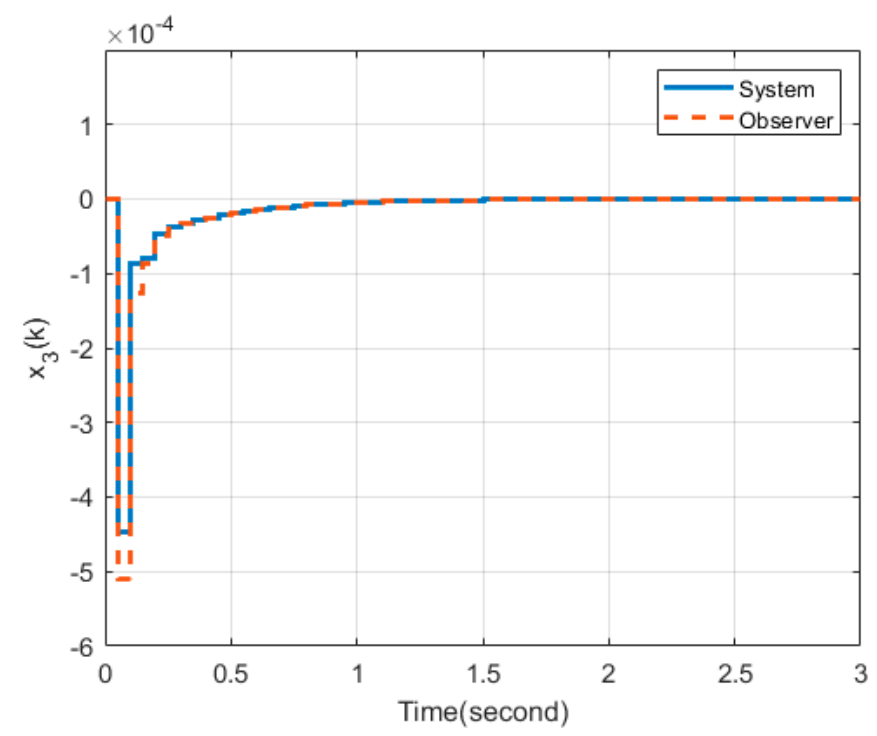

(c)

Figure 2. (a) The state and observer responses of $x_{1}(k)$; (b) The state and observer responses of $x_{2}(k)$; (c) The state and observer responses of $x_{3}(k)$.

The comparisons of state responses and estimation errors are shown in Figures 3 and 4, respectively. From Figure 3, it can be found that the proposed observerbased PD fuzzy control method has better state responses than the fuzzy control method investigated in [23]. Referring to Figure 4, the convergence of estimation errors of the proposed observer-based PD fuzzy control scheme is also faster than the method in [23]. It not only shows the superiority of the proposed design method but corresponds to that the PD feedback scheme can be reasonably used in the case of the high accuracy state observer. 


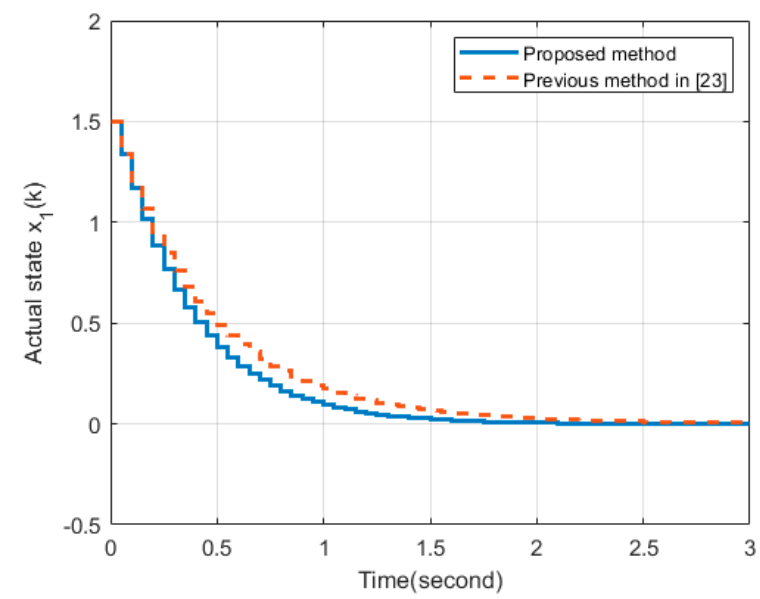

(a)

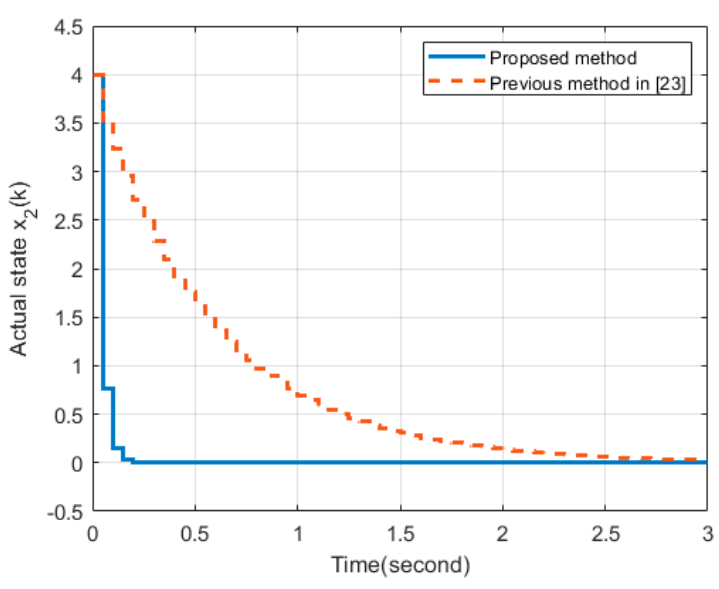

(b)

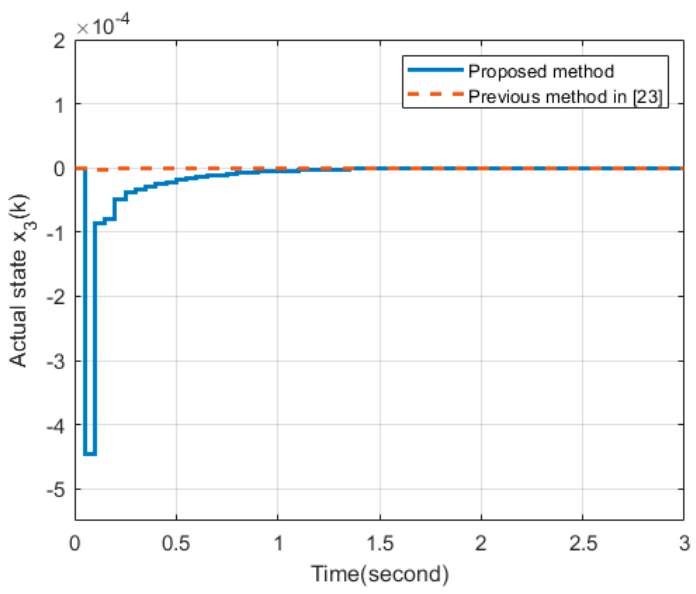

(c)

Figure 3. (a) The comparisons of $x_{1}(k)$; (b) The comparisons of $x_{2}(k)$; (c) The comparisons of $x_{3}(k)$.

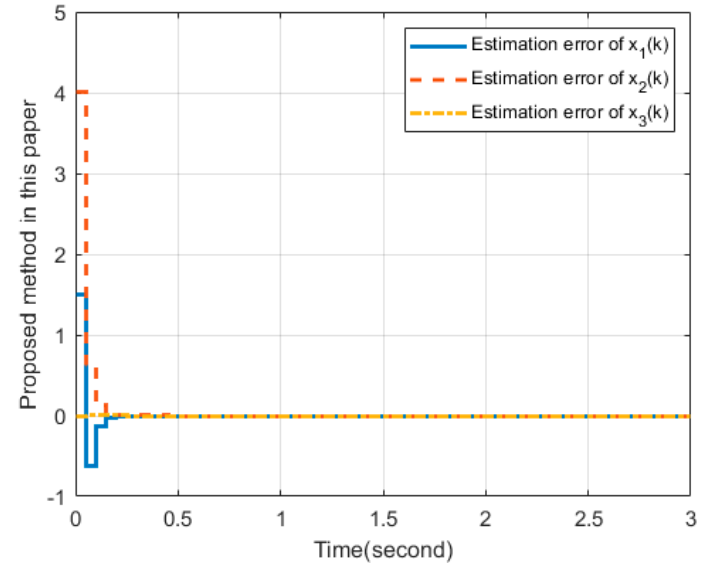

(a)

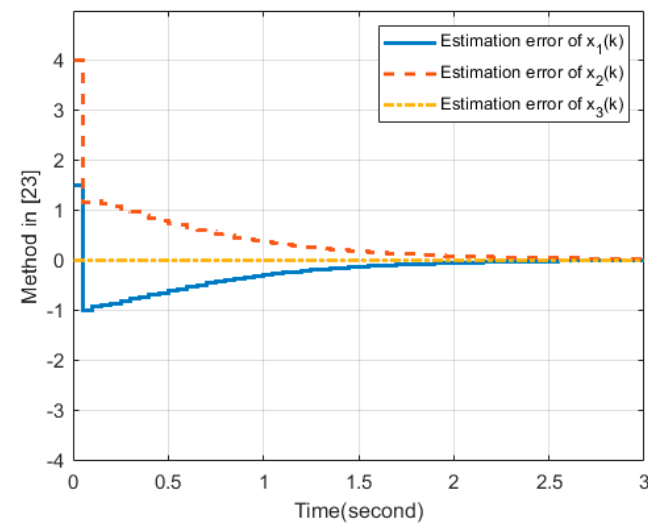

(b)

Figure 4. (a) Estimated state errors of the proposed design method; (b) Estimated state errors of the previous method [23]. 
The sensitivity and robustness analysis methods usually provide useful information to the development of control systems $[37,38]$. To enhance the robustness to disturbances and the sensitivity to faults, it is necessary to measure the robustness and sensitivity by a suitable performance index and optimize it. The robustness and sensitivity analysis of control systems with respect to the parametric variations of the controlled plant is not the major goal of this paper. However, it is worth mentioning that the robustness and sensitivity analysis methods $[37,38]$ could help designers extend the proposed control approach to investigate the robust fuzzy control for the perturbed discrete-time nonlinear singular systems.

\section{Conclusions}

In this paper, the observer-based PD feedback fuzzy control issue for the discretetime T-S fuzzy singular system has been studied. By applying the PD feedback scheme, the impulse terms generated from algebraic equations are eliminated, i.e., regularity and causality are naturally satisfied without any model transformations. Moreover, the PD fuzzy observer is constructed to solve the practical problems of unmeasured states and to satisfy the reasonable consideration of the PD controller. According to Lyapunov theory and transformation technologies, novel stability criteria in terms of LMI frameworks are developed. The effectiveness and advantage of the proposed method are verified with a numerical simulation. Due to packet loss problems and the disturbance effect, the present results could be extended to address the observer-based robust fuzzy control for the discrete-time T-S fuzzy singular systems with uncertainties. Furthermore, choosing a less conservative Lyapunov function is also an interesting problem for improving the proposed observer-based PD fuzzy control method.

Author Contributions: Conceptualization, W.-J.C.; methodology, W.-J.C. and M.-H.T.; software, M.-H.T.; validation, W.-J.C., M.-H.T. and C.-L.P.; investigation, M.-H.T. and C.-L.P.; writing-original draft preparation, W.-J.C. and M.-H.T.; writing-review and editing, W.-J.C. and M.-H.T. All authors have read and agreed to the published version of the manuscript.

Funding: This work was supported by the National Science Council of the Republic of China under Contract MOST109-2221-E-019-049.

Institutional Review Board Statement: Not applicable.

Informed Consent Statement: Not applicable.

Data Availability Statement: Not applicable.

Acknowledgments: The authors would like to express their sincere gratitude to the anonymous reviewers who gave us many constructive comments and suggestions. This work was supported by the National Science Council of the Republic of China under Contract MOST109-2221-E-019-049.

Conflicts of Interest: The authors declare no conflict of interest.

\section{References}

1. Qi, W.; Zong, G.; Karim, H.R. Observer-Based Adaptive SMC for Nonlinear Uncertain Singular Semi-Markov Jump Systems with Applications to DC Motor. IEEE Trans. Circuits Syst. I Regul. Pap. 2018, 65, 2951-2960. [CrossRef]

2. Bessaoudi, T.; Ben Hmida, F.; Hsieh, C. Robust state and fault estimation for linear descriptor stochastic systems with disturbances: A DC motor application. IET Control Theory Appl. 2017, 11, 601-610. [CrossRef]

3. Zhang, Q.; Liu, C.; Zhang, X. Complexity, Analysis and Control of Singular Biological Systems; Springer: London, UK, 2012; Volume 421.

4. Shi, P.; Wang, H.; Lim, C.-C. Network-Based Event-Triggered Control for Singular Systems With Quantizations. IEEE Trans. Ind. Electron. 2016, 63, 1230-1238. [CrossRef]

5. Wang, H.; Ying, Y.; Lu, R.; Xue, A. Network-based H $\infty$ control for singular systems with event-triggered sampling scheme. Inf. Sci. 2016, 329, 540-551. [CrossRef]

6. Xi, J.; Yu, Y.; Liu, G.; Zhong, Y. Guaranteed-Cost Consensus for Singular Multi-Agent Systems With Switching Topologies. IEEE Trans. Circuits Syst. I Regul. Pap. 2014, 61, 1531-1542. [CrossRef]

7. Sun, X.; Zhang, Q.; Yang, C.; Su, Z. Stability analysis and stabilization for discrete-time singular delay systems. J. Syst. Eng. Electron. 2011, 22, 482-487. [CrossRef] 
8. Sun, Y.; Ma, S. Observer-based finite-time stabilization for discrete-time switched singular systems with quadratically innerbounded nonlinearities. Int. J. Robust Nonlinear Control 2019, 29, 2041-2062. [CrossRef]

9. Fan, X.; Zhang, Q.; Ren, J. Event-triggered sliding mode control for discrete-time singular system. IET Control Theory Appl. 2018, 12, 2390-2398. [CrossRef]

10. Han, Y.; Kao, Y.; Gao, C. Robust observer-based H control for uncertain discrete singular systems with time-varying delays via sliding mode approach. ISA Trans. 2018, 80, 81-88. [CrossRef]

11. Chen, J.; Lin, C.; Chen, B.; Wang, Q.-G. Fuzzy-model-based admissibility analysis and output feedback control for nonlinear discrete-time systems with time-varying delay. Inf. Sci. 2017, 412-413, 116-131. [CrossRef]

12. Ma, Y.; Yang, P.; Zhang, Q. Robust $\mathrm{H} \infty$ control for uncertain singular discrete T-S fuzzy time-delay systems with actuator saturation. J. Frankl. Inst. 2016, 353, 3290-3311. [CrossRef]

13. Takagi, T.; Sugeno, M. Fuzzy identification of systems and its applications to modeling and control. IEEE Trans. Syst. Man Cybern. 1985, 1, 116-132. [CrossRef]

14. Precup, R.-E.; Preitl, S.; Petriu, E.; Bojan-Dragos, C.-A.; Szedlak-Stinean, A.-I.; Roman, R.-C.; Hedrea, E.-L. Model-based fuzzy control results for networked control systems. Rep. Mech. Eng. 2020, 1, 10-25. [CrossRef]

15. Stojčić, M.; Stjepanović, A.; Stjepanović, Đ. ANFIS model for the prediction of generated electricity of photovoltaic modules. Decis. Mak. Appl. Manag. Eng. 2019, 2, 35-48. [CrossRef]

16. Wang, H.O.; Tanaka, K.; Griffin, M.F. An approach to fuzzy control of nonlinear systems: Stability and design issues. IEEE Trans. Fuzzy Syst. 1996, 4, 14-23. [CrossRef]

17. Chang, W.-J.; Lin, Y.-H.; Du, J.; Chang, C.-M. Fuzzy Control with Pole Assignment and Variance Constraints for Continuous-time Perturbed Takagi-Sugeno Fuzzy Models: Application to Ship Steering Systems. Int. J. Control Autom. Syst. 2019, 17, $2677-2692$. [CrossRef]

18. Chang, W.-J.; Chang, C.-M.; Lin, Y.-H.; Du, J. Discrete-time robust fuzzy control synthesis for discretized and perturbed ship fin stabilizing systems subject to variance and pole location constraints. J. Mar. Sci. Technol. 2021, 26, 201-215. [CrossRef]

19. Ku, C.C.; Huang, P.H.; Chang, W.J. Passive fuzzy controller design for perturbed nonlinear drum-boiler system with multiplicative noise. J. Mar. Sci. Technol. 2010, 18, 211-220.

20. Yang, P.; Ma, Y.; Yang, F.; Kong, D.; Wang, L. Passivity control for uncertain singular discrete T-S fuzzy time-delay systems subject to actuator saturation. Int. J. Syst. Sci. 2018, 49, 1627-1640. [CrossRef]

21. Wang, Y.; Xia, Y.; Shen, H.; Zhou, P. SMC Design for Robust Stabilization of Nonlinear Markovian Jump Singular Systems. IEEE Trans. Autom. Control 2017, 63, 219-224. [CrossRef]

22. Wang, Y.; Karimi, H.R.; Lam, H.-K.; Yan, H. Fuzzy Output Tracking Control and Filtering for Nonlinear Discrete-Time Descriptor Systems Under Unreliable Communication Links. IEEE Trans. Cybern. 2020, 50, 2369-2379. [CrossRef] [PubMed]

23. Wu, J.; Fu, Q.; Li, Z. Control via state observer feedback for the T-S fuzzy singular system. Int. J. Mach. Learn. Cybern. 2017, 8, 619-626. [CrossRef]

24. Park, I.S.; Kwon, N.K.; Park, P. Dynamic output-feedback control for singular T-S fuzzy systems using fuzzy Lyapunov functions. Nonlinear Dyn. 2019, 98, 1957-1971. [CrossRef]

25. Gao, Z.; Liu, Y.; Wang, Z. On Stabilization of Linear Switched Singular Systems via P-D State Feedback. IEEE Access 2020, 8 , 97007-97015. [CrossRef]

26. Gao, Z.; Wang, Z.; Ji, Z.; Liu, Y. Output Strictly Passive Control of Discrete-Time Linear Switched Singular Systems Via Proportional Plus Derivative State Feedback. Circuits Syst. Signal Process. 2020, 39, 3907-3924. [CrossRef]

27. Kuo, Y.-C.; Lin, W.-W.; Xu, S.-F. Regularization of Linear Discrete-Time Periodic Descriptor Systems by Derivative and Proportional State Feedback. SIAM J. Matrix Anal. Appl. 2004, 25, 1046-1073. [CrossRef]

28. Bunse-Gerstner, A.; Nichols, N.K.; Mehrmann, V. Regularization of Descriptor Systems by Derivative and Proportional State Feedback. SIAM J. Matrix Anal. Appl. 1992, 13, 46-67. [CrossRef]

29. Ku, C.-C.; Chang, W.-J.; Tsai, M.-H.; Lee, Y.-C. Observer-based proportional derivative fuzzy control for singular Takagi-Sugeno fuzzy systems. Inf. Sci. 2021. [CrossRef]

30. Chang, W.; Su, C.; Varadarajan, V. Fuzzy controller design for nonlinear singular systems with external noises subject to passivity constraints. Asian J. Control 2020. [CrossRef]

31. Mu, Y.; Zhang, H.; Sun, S.; Ren, J. Robust non-fragile proportional plus derivative state feedback control for a class of uncertain Takagi-Sugeno fuzzy singular systems. J. Frankl. Inst. 2019, 356, 6208-6225. [CrossRef]

32. Mu, Y.; Zhang, H.; Su, H.; Zhang, K. Observer-based actuator fault estimation and proportional derivative fault tolerant control for continuous-time singular systems. Optim. Control Appl. Methods 2019, 40, 979-997. [CrossRef]

33. Ren, J.; Li, X.; Mu, Y. Proportional-Difference Observer Design for Singular Systems in an LMI Framework. IEEE Access 2020, 8 , 162449-162457. [CrossRef]

34. Apkarian, P.; Tuan, H.D.; Bernussou, J. Continuous-time analysis, eigenstructure assignment, and H/sub 2/ synthesis with enhanced linear matrix inequalities (LMI) characterizations. IEEE Trans. Autom. Control 2001, 46, 1941-1946. [CrossRef]

35. Xie, W.-B.; Li, H.; Wang, Z.-H.; Zhang, J. Observer-based Controller Design for A T-S Fuzzy System with Unknown Premise Variables. Int. J. Control Autom. Syst. 2019, 17, 907-915. [CrossRef]

36. Boyd, S.; El Ghaoui, L.; Feron, E.; Balakrishnan, V. Linear Matrix Inequalities in System and Control Theory; Society for Industrial and Applied Mathematics: Philadelphia, PA, USA, 1994. 
37. Qian, G. Sensitivity and Robustness in Industrial Engineering: Methodologies and Applications to Crash Tests, Mechanics; Université de Lyon: Lyon, France, 2017.

38. Pospíšil, J.; Sobotka, T.; Ziegler, P. Robustness and sensitivity analyses for stochastic volatility models under uncertain data structure. Empir. Econ. 2018, 57, 1935-1958. [CrossRef] 